\title{
Use of A Jig to Control Forearm Rotation and Wrist Position for MR and CT Imaging of Patients with Distal Radioulnar Joint and Wrist Dysfunction
}

\author{
Malone PSC ${ }^{1,2}$, Bhatti WA ${ }^{3}$ and Lees VC ${ }^{2 *}$ \\ ${ }^{1}$ Blond Mclndoe Laboratories, University of Manchester, UK \\ ${ }^{2}$ Department of Plastic Surgery, University Hospital South Manchester, Wythenshawe, UK \\ ${ }^{3}$ Department of Radiology, University Hospital South Manchester, Wythenshawe, UK
}

*Corresponding author: VC Lees, Department of Plastic Surgery, University Hospital South Manchester, Wythenshawe Hospital,

Southmoor Road, Manchester M23 9LT, United Kingdom

\section{ARTICLE INFO}

Received: 蔧 August 26, 2021

Published: 幽 September 07, 2021

Citation: Malone PSC, Bhatti WA, Lees VC. Use of A Jig to Control Forearm Rotation and Wrist Position for MR and CT Imaging of Patients with Distal Radioulnar Joint and Wrist Dysfunction. Biomed J Sci \& Tech Res 38(4)-2021. BJSTR. MS.ID.006183.

Keywords: The rule of choice of equations for $\mathrm{K}_{\mathrm{i}}$ and $\mathrm{K}_{\mathrm{a}}$ constants calculation

\section{ABSTRACT}

Use of a jig to control forearm rotation and wrist position for MR and CT imaging of patients with distal radioulnar joint and wrist dysfunctiong.

Aim: To design and assess efficacy of a jig to enhance MR and CT imaging of the distal forearm.

Materials and Methods: A forearm-support jig was developed for clinical use in imaging the forearm, DRUJ, TFCC and wrist joint. The jig was constructed in MRl- safe materials and incorporated a goniometer allowing control of forearm rotation and a support for wrist position. The jig was used as an adjunct to a clinical research project on the DRUJ both in patients and volunteers. For the purpose of assessment of the utility of the jig record was made of patient comfort and acceptability, end- image quality output, reproducibility of scans repeated intra- and inter-subject, and ease of use by the radiographers.

Results: Twenty-three volunteers found the jig to be comfortable during the scanning procedure. Nine patients who had also undertaken standard MRl scans without the jig unanimously reported improved comfort and satisfaction levels with the jig. Radiographers found the device easy to use obviating the need to repeat scans due to patient movement or incorrect wrist positioning. lmages obtained readily permitted intra- and inter-patient comparison. lmage quality was improved with less subject tremor.

Conclusion: A jig has been developed that allows standardization of position of forearm rotation. This has proved a useful adjunct for MR and CT imaging of the forearm and DRUJ where the position of forearm rotation needs to be controlled for the purpose of the study or investigation.

\section{Introduction}

The distal radioulnar joint (DRUJ), triangular fibrocartilaginous complex (TFCC) and the forearm are frequently imaged by MR and CT for associated disease processes. The standard 'superman' position is often uncomfortable for patients generating movement artefact. Scans are restricted to the prone position of forearm rotation placing potential limitation on the information that might be gained from viewing the same structures in different positions of forearm rotation. For example, a tear of the volar distal radioulnar 
ligament is easily identified in supination with dorsal subluxation of the distal radius on the ulnar head. However, the diagnosis can be easy to miss with the forearm pronated, where the subluxation is less evident.

Patient discomfort particularly in MRl scanning is significant even with modern generation scanners. Patients must remain as still as they can whilst maintaining the body prone position with extended shoulder and elbow and forearm pronated. Upper limb support is improvised padding if present.

Gross movement blurs images and fine tremor artefact occurs with the strain of trying to maintain perfectly still. This is particularly pertinent with the current use of high-Tesla scanners. Whilst such scanners offer higher definition imaging unwanted effects from tremor and movement are more troublesome. In specific research studies, lack of standardization of forearm positioning creates systematic error with respect to intra- and inter-patient comparisons. Even comparison between serial scans in the same individual can be challenging without ensuring an identical posture is maintained. This study reports the development and trialing of a forearm jig used in a research study on soft tissues of the distal forearm, reported elsewhere [1]. A scanning platform was developed for this purpose, with the aim of allowing both clear visualization of key soft tissue structures about the wrist using a 3-Tesla MRl machine, as well as permitting both intra- and interpatient comparability of scans.

\section{Methods}

A scanning-platform jig was designed solely from radio-opaque materials, suitable for use with both CT and MRl scanners (Figure 1). Two padded straps fasten either side of the biceps, removing the effect of shoulder movements. A third strap holds the wrist to a padded hand platform. In this manner, all rotatory movements are isolated to the radius and ulna. The antecubital fossa faces superiorly. The fingers and thumb rest on a palmar support of adjustable height to provide approximately 20 degrees dorsiflexion and 15 degrees ulna deviation throughout the scanning process. The wrist posture can be adjusted as needed. The jig is adaptable to different habitus and patient position. The platform of the jig permits rotation and incorporates a goniometer together with a long handle for rotation that can be adjusted mid-scan by the radiologist without needing to remove the patient from the scanner. Registration markers are incorporated into the scanning platform to orientate the radiologist. Standard MR wrist coils can be placed adjacent to wrist and forearm and function normally. The construct is simple to clean.

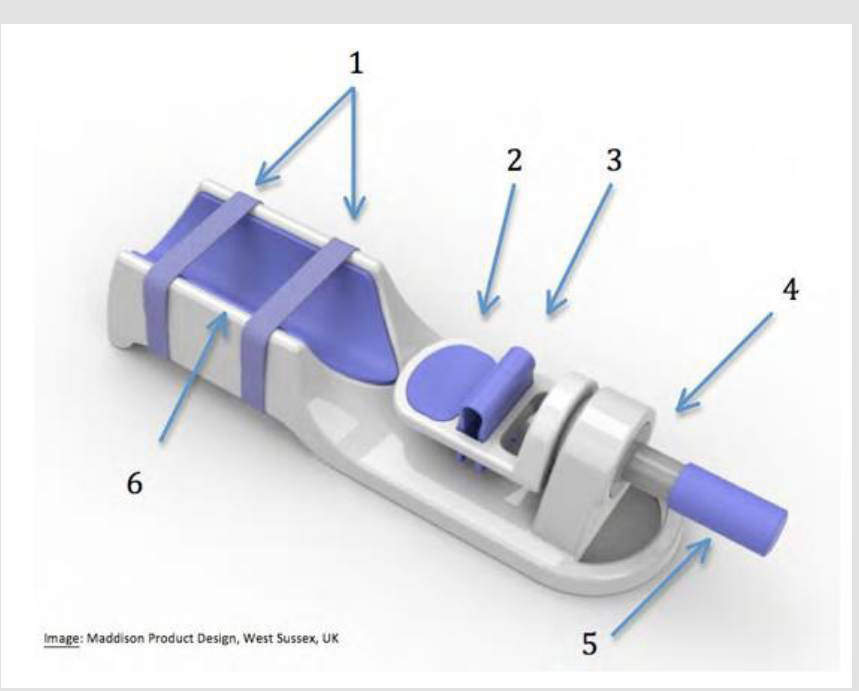

Figure 1: Features of the scanning jig.

1. Upper arm straps

2. Platform for palm of hand

3. Adjustable height gripping block

4. Incorporated goniometer

5. Platform rotation handle

6. Upper arm rest.

The use of disposable 'arm gloves' where needed reduces risk of cross-contamination. Patients and volunteers were recruited for a research study imaging soft tissue structures in the distal forearm. The forearm jig was used to undertake a sequence of scans on one upper limb at a time on a 3-Tesla MRl scanner. Patients were symptomatic of DRUJ dysfunction, whilst volunteers 
were required to have no history of prior upper limb trauma and a normal clinical examination. An assessment of the subject's range of pronosupination was made to inform the limits of rotation. Each arm was scanned in 5 positions of pronosupination (maximal supination - SMax; 30 degrees supination - S30; neutral;
30 degrees pronation - P30; maximal pronation - PMax) using a prototype of the scanning-platform-jig as illustrated (Figure 2). lnformed consent was obtained from all recruited individuals: Ethical permission was granted by the UK National Research Ethics Committee [Reference number 07/Q1406/7].

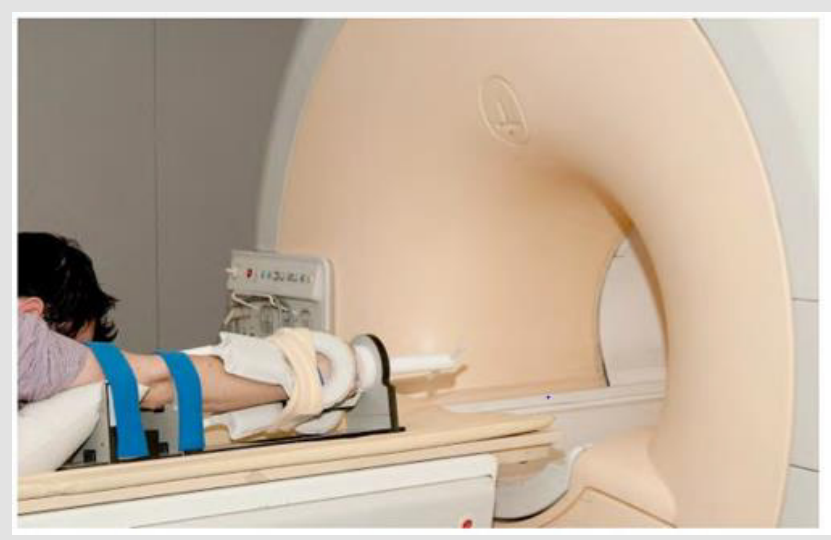

Figure 1: Jig prototype in use in an MRI machine with wrist coils attached.

Following the scans all subjects were asked if they experienced any discomfort during the scan related to the jig. Patients who had prior experience of conventional wrist MR scan were asked how it compared to their previous experience. Operators were asked to comment on how difficult they found the jig to use and what their thoughts were of the impact upon the scanning process. Opensource image processing and DICOM workstation software, OsiriX ${ }^{\circledR}$ v3.1 32- bit (OsiriX Foundation, Geneva, Switzerland) was used to compare corresponding image series of left and right wrists: lmages were co-registered by identifying the ulna fovea and then sequences synchronized for scrolling purposes. Those of the left wrist were then flipped $180^{\circ}$ horizontally to allow direct side-byside comparison between left and right. The first author analyzed the scans together with a consultant radiologist: lntra- subject comparability was assessed by establishing the orientation of the bony structures about the DRUJ and then determining if both left and right wrists were in the same position of pronosupination; lnter-subject comparability was assessed for in a blinded manner by looking at individual scan slices including the ulna fovea or ulna styloid, and recording whether it was believed that the scans were taken in SMax, S30, neutral, P30 or PMax. Further assessment was made to verify if the images for each position of pronosupination were comparable across all of the wrists scanned.

The results were collated and comments noted.

\section{Results}

Thirty-three subjects were recruited for the scans: 9 patients (mean age 41 years; range 27-55) and 24 volunteers (mean age 34 years; range 19-57). One-person experienced claustrophobia and the scan was aborted. With the exception of a patient who had metalwork in one wrist, both wrists were scanned in 5 positions for each individual, giving a total of 10 scan series per patient, and an overall dataset of 315 scan series. Due to a variety of image sequences being used, the time taken to scan each wrist was about 50 minutes. A single series contained 40 images at $1 \mathrm{~mm}$ intervals through the DRUJ and took approximately 13 minutes to obtain using our 3Tesla machine. All scans were performed in body prone arm extended position. All 32 subjects reported the jig as comfortable. All 9 patients who had undertaken previous wrist MR scans said that they preferred the scans using the jig than without because it felt more comfortable. In particular, a constant factor was that patients stated that they could better relax because their arm was strapped-in and so they didn't need to worry about it moving or actively try to keep it completely still.

Seven people were so relaxed they fell asleep. Four of the first 10 subjects scanned reported slight discomfort using the jig for so long: this was overcome by adjustment of the padding provided. Staff found the jig simple to use. It was reported as 'easy' to strap the patient/volunteer into the jig and to arrange their upper limb into the exact position required. Application of wrist coils whilst the hand was strapped onto the jig was straightforward. Mid-scan, wrist position was easily adjusted by the radiographer without needing to remove the patient from the scanner tube. This reduced scan time by removing the need for further registration/ location scans. No scans needed to be repeated due to patient movement. Overall, radiographers stated that there was a faster, more predictable speed of throughput of patients and volunteers whilst using the jig than they had previously experienced in upper limb 
scans without using any such device. For image analysis, positions of forearm rotation matched for both intra- (31/31) and inter$(63 / / 63)$ subject comparisons in $100 \%$ of wrists for S30, neutral and P30.

The only instances whereby left and right wrists did not closely match for intra-subject variability were for those individuals whose SMax and PMax values were different. This occurred in our patient cohort where symptomatic wrists demonstrated some reduction of range of rotation. Nonetheless, it was still correctly reported (31/31) by the interpreter that of the choices given, these scans were done at the extremes of the individual's range of rotation. For intersubject variability with SMax and PMax, due to natural variation in the permissible range of pronosupination in the normal population, there were expected differences in degree of rotation observed. However, the only instances where scans were questioned as to whether or not of the 5 given positions they were at the extreme of rotation, were in 2 patients who each had a dramatically reduced SMax (42 \& 44 degrees): therefore only 61/63 scans were correctly identified as being in SMax. Despite the lengthy scan time, only slight patient tremor occurred. None of the 315 scan series needed repeat scanning on account of movement artefact.

\section{Discussion}

Forearm pronosupination and wrist position affect the relationships of the key Osseo ligamentous structures. Standardization and control of forearm position should help imaging and enhance diagnosis of pathology related to the ligamentous structures and also assist intra- and inter-patient comparison. It is perhaps surprising that MR and CT imaging protocols have not routinely included means of standardizing forearm rotation and wrist position. Certain conditions such as tears of the volar part of the TFCC complex will be better visualized in forearm supinated position and are potentially under-diagnosed with the forearm routinely placed in pronation. Conventional limb positioning has been described [2] including the pronated position for upper limb radiographs [3-5]. The optimal imaging and sequencing techniques for investigating structures such as the DRUJ and TFCC are still a matter of debate with various opinions expressed [6-9]. Developing a means by which to allow intra- and inter-individual comparability between MR scans was not without limitation.

There was no pre-existing system for comparison to build upon therefore a logical method was developed to do this by inferring the position of pronosupination in reference to key constant landmarks, notably the orientation of the ulna styloid and ulna fovea. The jig minimized patient movement artefact but did not completely eliminate fine tremor impacting final image quality. Nonetheless, it is important to improve patient comfort and select forearm position during these scans as the gains from higher resolution machines will not be realized if patient position and movement cannot be controlled.

\section{Conclusion}

A forearm and wrist platform-support jig has been developed and the utility of the jig has been demonstrated in a study looking at the effect of forearm rotation on soft tissues. Use of the jig produced high quality images over relatively long scan times minimizing movement artefact. Control of forearm position may be important in imaging for disorders of forearm rotation. The authors have found the jig to be of particular use in the research setting but believe there are clinical applications in the assessment of ulnarsided wrist pain.

\section{Conflict of Interest Statement}

The device as described in the current paper is registered for further development and distribution through UMIP at the University of Manchester. Any future royalties from successful distribution of this jig would be allocated to research funds.

\section{Funding}

This research was supported by funding from the Stanley Thomas Johnson Foundation, University of Manchester MRC Interdisciplinary Bridging Award administered through the Manchester centre for the Integration of Medicine and lnnovative Technology (MIMIT), the Transitional Imaging Unit (TlU), Salford Royal Hospital NHS Foundation Trust, UK and UMIP, the University of Manchester's Department of Intellectual Property, UK.

\section{Acknowledgement}

- Mr R Williams, former Clinical Engineer, University Hospital South Manchester, UK

- Mr R Hanmer and Mr D Harrison, Department of Medical Engineering, Salford Royal Hospital NHS Foundation Trust, UK

- $\quad$ Professor P Williams, Research \& Development Lead, Christie Hospital.

- $\quad$ TIU departmental team, Transitional lmaging Unit, Salford Royal Hospital NHS Foundation Trust, UK

- UMIP, University of Manchester Intellectual Property team, University of Manchester, UK.

\section{References}

1. Malone PSC, Hutchinson CE, Kalson NS, Twining CJ, Terenghi G, et al. (2011) Subluxation-related ulnar neuropathy (SUN) syndrome related to distal radioulnar joint instability. Journal of Hand Surgery 37(7): 65264.

2. Steinbach LS, Smith DK (2000) MRI of the wrist. Clin Imaging 24(5): 298-322. 
3. Epner RA, Bowers WH, Guilford WB (1982) Ulnar variance--the effect of wrist positioning and roentgen filming technique. J Hand Surg 7(3): 298-305.

4. Yeh GL, Beredjiklian PK, Katz MA, Steinberg DR, Bozentka DJ (2001) Effects of forearm rotation on the clinical evaluation of ulnar variance. J Hand Surg Am 26(6): 1042-1046.

5. Palmer AK, Glisson RR, Werner FW (1982) Ulnar variance determination. J Hand Surg 7(4): 376-379.

6. Maizlin ZV, Brown JA, Clement JJ, Donna E Smith, Jon A Jacobson, et al. (2009) MR Arthrography of the Wrist: Controversies and Concepts. HAND 4(1): 66-73.

ISSN: 2574-1241

DOI: 10.26717/BJSTR.2021.38.006183

Lees VC. Biomed J Sci \& Tech Res

(C) This work is licensed under Creative

Submission Link: https://biomedres.us/submit-manuscript.php
7. Haims AH, Schweitzer ME, Morrison WB (2002) Limitations of MR imaging in the diagnosis of peripheral tears of the triangular fibrocartilage of the wrist. AJR American journal of roentgenology 178(2): 419-422.

8. Hobby JL, Tom BD, Bearcroft PW, Dixon AK (2001) Magnetic resonance imaging of the wrist: diagnostic performance statistics. Clinical radiology 56(1): 50-57.

9. Meier R, Schmitt R, Krimmer H (2005) [Wrist lesions in MRI arthrography compared with wrist arthroscopy]. Handchir Mikrochir Plast Chir 37 (2): 85-89.

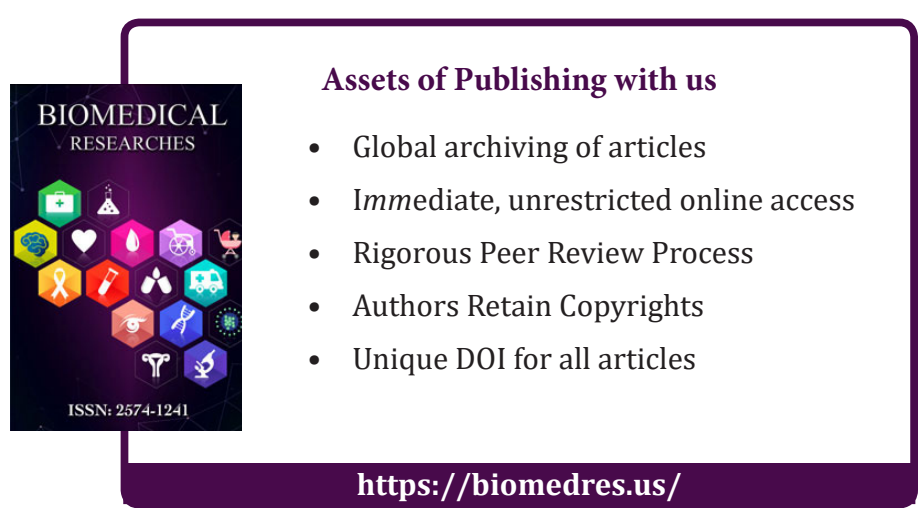

